\title{
Performance Analysis of Repeated Index Modulation with Coordinate Interleaving over Nakagami- $m$ Fading Channel
}

\author{
Le Thi Thanh Huyen, Tran Xuan Nam \\ Le Quy Don Technical University, Hanoi, Vietnam \\ Correspondence: Le Thi Thanh Huyen, huyen.ltt@mta.edu.vn \\ Communication: received 17 May 2019, revised 21 June 2019, accepted 22 June 2019 \\ Online early access: 25 June 2019, Digital Object Identifier: 10.32913/mic-ict-research.v2019.n1.863 \\ The Area Editor coordinating the review of this article and deciding to accept it was Dr. Truong Trung Kien
}

\begin{abstract}
In this paper, a new Space-Time Block Coded Spatial Modulation (SM) scheme based on the Golden Code, called the In this paper, we evaluate the symbol error performance of an extended Index Modulation for Orthogonal Frequency Division Multiplexing (IM-OFDM), namely repeated index modulation-OFDM with coordinated interleaving (abbreviated as ReCI), over the Nakagami- $m$ fading channel. The ReCI system attains higher error performance than the conventional IM-OFDM with coordinate interleaving (IMOFDM-CI). In order to investigate the system performance over the Nakagami- $m$ fading channel, we derive the closedform expressions for the symbol error probability (SEP) and the bit error probability (BEP). The analytical results give interesting insights into the dependence of SEP on system parameters. Their tightness is also validated by numerical results, which show that our proposed scheme can provide considerably better error performance than the conventional IM-OFDM and IM-OFDM-CI at the same spectral efficiency.
\end{abstract}

Keywords: Index modulation, IM-OFDM, coordinate interleaving, pair-wise error probability, symbol error probability, maximum likelihood detection, Nakagami-m fading.

\section{INTRODUCTION}

Index modulation for orthogonal frequency division multiplexing (IM-OFDM) has emerged as an effective index modulation scheme in the frequency domain as it achieves not only higher energy efficiency but also better error performance than the conventional OFDM. The IM-OFDM system activates only a subset of sub-carriers for transmission. In comparison with the classical OFDM, the IMOFDM system uses both the $M$-ary modulated symbols and indices of active sub-carriers to convey information bits.

In the early IM-OFDM system [1], a fixed number of information bits were employed to activate the subcarriers, thus its spectral efficiency and error performance were limited. In order to overcome this disadvantage, an IM-OFDM scheme with adjustable active sub-carriers according to incoming bits was proposed in [2]. In another effort, the sub-carriers were interleaved in [3] to extend the Euclidean distance among the complex data symbols to deal with the error performance issue. Moreover, the study in [4] proposed the interleaved sub-carrier grouping and investigated the achievable rate of the system.

Regarding the spectral efficiency issue, the IM-OFDMI/Q scheme in [5] performed joint index modulation over both the in-phase and quadrature components of the $M$ ary modulated symbols. In another solution, the dual-mode OFDM (DM-OFDM) was introduced in [6]. This model utilized inactive sub-carriers to carry more data bits together with active sub-carriers. Different signal constellations were used for complex data symbols over the active and inactive sub-carriers. The work in [7] introduced a multimode IM-OFDM (MM-IM-OFDM) scheme which used all sub-carriers and extra information bits were conveyed by permutation of transmission modes, thus attaining further increase in spectral efficiency. In order to achieve not only spectral efficiency but also diversity gain, the authors in [8] successfully designed a linear constellation precoder (LPC) for IM-OFDM. The recent study in [9] attained an improvement for both diversity gain and energy efficiency by applying compressed sensing to IM-OFDM.

Meanwhile, there were also a variety of researches focused on analyzing the performance of IM-OFDM. The authors in [10] provided a frame work for deriving a tight bound for BER of IM-OFDM. The work [11] evaluated the outage probability of the IM-OFDM system over two-way diffused-power fading channels. The transmission reliability of IM-OFDM and IM-OFDM employing greedy detection under imperfect channel state information (CSI) were 
investigated through SEP in [12] and [13], respectively. Besides, an opportunistic scheduling IM-OFDM (OS-IMOFDM) scheme which exploits multi-user diversity gain to improve the system performance of IM-OFDM is reported in [14]. Diversity and coding gains of OS-IM-OFDM and OS-OFDM were also compared in this study.

Focusing on diversity gain issue, the coordinate interleaved IM-OFDM scheme in [15] distributed the real and imaginary components of the $M$-ary modulated data symbols over distinctive sub-carriers. The paper [16] introduced an IM-OFDM scheme with transmit diversity, which utilized multiple signal constellations to carry the same data bits over the active sub-carriers. In the recent work [17], the coded IM-OFDM with transmit diversity (TD-IM-OFDM) was proposed to increase the reliability for index detection. The authors of [18] introduced a novel spread IM-OFDM scheme to improve the transmit diversity and low-complexity detectors were also designed in this work. To further improve the diversity gain and error performance, the IM-OFDM concept was extended to MIMO systems in $[19,20]$. In order to reduce complexity while still improving the diversity gain of IM-OFDM, the study in [21] introduced the IM-OFDM with greedy detection and diversity reception. Its BER performance under imperfect CSI was analyzed in [22]. The repeated IM-OFDM with transmit diversity (ReMO) and the closed-form expressions for its SEP and BER were presented in [23].

Recently, there was a proposal to apply deep learning to detect data bits of the IM-OFDM systems in [24]. The proposed deep-learning assisted detector could provide a near optimal performance as the ML and considerably reduces the runtime of existing detectors.

Aiming at improving the error performance of the conventional IM-OFDM-CI system, we proposed a repeated index modulation-OFDM with coordinated interleaving scheme in [25], which is abrreviated as ReCI. In this scheme, coordinate interleaving is simultaneously applied to the $M$-ary modulated symbols in two different clusters. Additionally, these distinct clusters repeatedly utilize the same set of active sub-carrier indices. Combining coordinate interleaving and the index repetition allows ReCI to attain better index detection and the $M$-ary modulated symbol error performance over the conventional IM-OFDM and IM-OFDM-CI systems, even at the same spectral efficiency. Furthermore, while IM-OFDM-CI requires an even number of active sub-carriers, our proposed scheme can work well with an arbitrary number of sub-carriers and thus is more flexible in terms of achieving error performance and spectral efficiency. However, this work lacks theoretical analysis on SEP and BEP performance. Additionally, the impacts of system parameters on the transmission reliability were also not properly analyzed. Besides, most previous IM-OFDM studies only investigated the system performance over the Rayleigh fading channel. For realistic conditions, received signal intensity follows the Nakagami- $m$ distribution, which is more suitable for multipath channels than the Rayleigh. The Nakagami- $m$ fading channel is most suitable for the urban, suburban, as well as both the indoor and outdoor propagation model [26].

In this paper, we analyze performance of ReCI in terms of SEP and BEP. The analytical results allow us to obtain an helpful insight into the impact of system parameters on the error performance. Thus, it is possible for us to design a system configuration with the best error performance.

The remainder of this paper is organized as follows. Section II describes the system model of the proposed ReCI. The error performance is analyzed in Section III. Simulation results and discussions are given in Section IV. Finally, the conclusion is given in Section V.

Notation: Vectors and matrices are denoted by bold letters. $C(.,$.$) and \lfloor\cdot\rfloor$ present the binomial coefficient and the floor function, respectively. $\mathbb{E}\{\cdot\}$ is the expectation operation. $(\cdot)^{R}$ and $(\cdot)^{I}$ denote the real and imaginary parts of a complex number, respectively. The moment generating function is denoted as $\mathcal{M}(\cdot)$.

\section{SySTEM MOdeL}

The block diagram of one ReCI sub-block is depicted in Figure 1. Assume that the system with $N_{t}$ sub-carriers is split into $G$ sub-blocks of $N_{G}$ sub-carriers, i.e., $N_{t}=G N_{G}$. Then, each sub-block is partitioned into two clusters of $N$ sub-carriers, i.e., $N_{G}=2 N$. Since the operation in each sub-block is independent, without loss of generality, we consider only one sub-block.

In each sub-block transmission, $p$ incoming bits are divided into two bit sequences. The first $p_{1}=$ $\left\lfloor\log _{2}(C(N, K))\right\rfloor$ bits are sent to an index selector to determine $K$ out of $N$ sub-carriers for each cluster, using either a look-up table (LUT) or the combinatorial method [2]. Similar to the IM-OFDM system, an additional number of information bits is transfered through the indices of active sub-carriers. The remaining $N-K$ sub-carriers are set to zero. The output of the index selector is a set of activated sub-carrier indices $\theta$, i.e., $\theta=\left\{\alpha_{1}, \ldots, \alpha_{K}\right\}$, where $\alpha_{k}=\{1, \ldots, N\}$ and $k=\{1, \ldots, K\}$. Let $\mathcal{I}$ denote the set of possible active indices. For a given $N$ and $K$, there are a total of $c=2^{\left\lfloor\log _{2} C(N, K)\right\rfloor}$ combinations of active indices. Different from the conventional IM-OFDMCI, ReCI employs the same set of active sub-carrier indices $\theta$ for two clusters in one sub-block as illustrated in Figure 1. It is noteworthy that such index repetition can improve the 


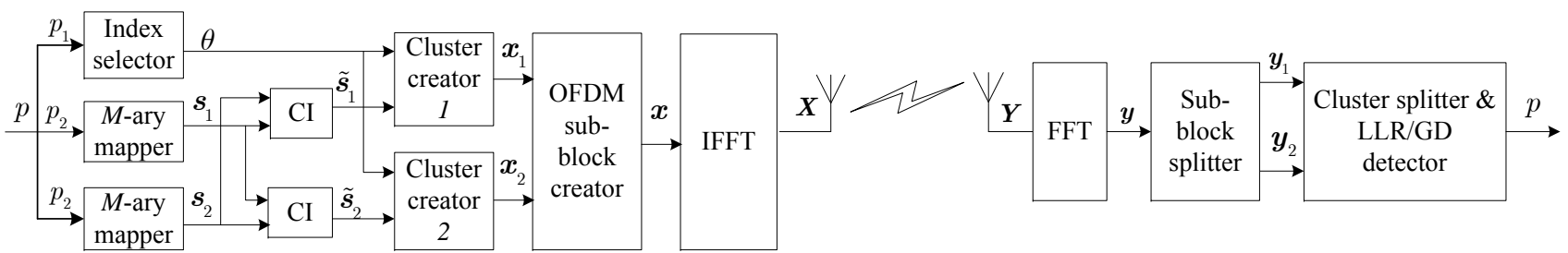

Figure 1. Block diagram of one ReCI sub-block.

index error probability (IEP) over the conventional scheme at the cost of spectral efficiency.

The second bit sequence is equally separated into two clusters of $p_{2}=K \log _{2} M$ bits. By repeating the indices of sub-carriers between two clusters, we can jointly apply the coordinate interleaving technique to the $M$-ary modulated symbols from two distinguishable clusters. For cluster $i$, $p_{2}=K \log _{2} M$ bits are mapped into a vector of $K M$ ary modulated symbols $\mathbf{s}_{i} \in \mathbb{C}^{K \times 1}$, for $i=1,2$. Let $\mathbf{s}_{1}=\left[\begin{array}{llll}a_{1} & a_{2} & \ldots & a_{K}\end{array}\right]^{T}$ and $\mathbf{s}_{2}=\left[\begin{array}{llll}b_{1} & b_{2} & \ldots & b_{K}\end{array}\right]^{T}$. Prior to the coordinate interleaving, all $M$-ary modulated symbols in $\mathbf{s}_{i}$ are rotated by an angle $\phi$ which is defined based on the modulation type. Let $\mathcal{S}^{\phi}$ denote the rotated constellation. The values of $\phi$ can be selected by the computer search method [15]. For example, the quadrature amplitude modulation (QAM) with constellation size $M=\{2,4,16,64\}$, rotation angles are respectively given by $\phi=\left\{45^{\circ}, 15^{\circ}, 8.5^{\circ}, 4.5^{\circ}\right\}$. After coordinate interleaving between the symbols in $\mathbf{s}_{1}$ and $\mathbf{s}_{2}$ from different clusters, the symbol vectors in each cluster, $\tilde{\mathbf{s}}_{i} \in \mathbb{C}^{K \times 1}$, are obtained as follows:

$$
\begin{gathered}
\tilde{\mathbf{s}}_{1}=\left[\begin{array}{c}
c_{1,1} \\
c_{1,2} \\
\vdots \\
c_{1, K-1} \\
c_{1, K}
\end{array}\right]=\left[\begin{array}{c}
a_{1}^{R}+j b_{1}^{I} \\
a_{2}^{R}+j b_{2}^{I} \\
\vdots \\
a_{K-1}^{R}+j b_{K-1}^{I} \\
a_{K}^{R}+j b_{K}^{I}
\end{array}\right], \\
\tilde{\mathbf{s}}_{2}=\left[\begin{array}{c}
c_{2,1} \\
c_{2,2} \\
\vdots \\
c_{2, K-1} \\
c_{2, K}
\end{array}\right]=\left[\begin{array}{c}
b_{1}^{R}+j a_{1}^{I} \\
b_{2}^{R}+j a_{2}^{I} \\
\vdots \\
b_{K-1}^{R}+j a_{K-1}^{I} \\
b_{K}^{R}+j a_{K}^{I}
\end{array}\right] .
\end{gathered}
$$

where $j=\sqrt{-1}$. Using $\tilde{\mathbf{s}}_{i}$ and $\theta$, the transmitted codeword over $N$ sub-carriers $\mathbf{x}_{i}=\left[x_{i 1}, \ldots, x_{i N}\right]^{T}$ is generated. Since only $K$ out of $N$ sub-carriers are activated, $K$ symbols corresponding to $K$ active sub-carriers are nonzero, i.e., $x_{i \alpha_{k}}=c_{i, k}$ when $\alpha_{k} \in \theta$, and $x_{i \alpha}=0$ when $\alpha \notin \theta$, where $\alpha_{k}=\{1, \ldots, N\}, k=\{1, \ldots, K\}, i=1,2$. An example of a lookup table to determine the transmitted codewords in each cluster when $N=4, K=2, p_{1}=2$ is presented in Table I. It can be seen that for each data symbol in a cluster, its real and imaginary components are transferred over different sub-carriers. In addition, combining the index repetition and the joint coordinate interleaving allows $\mathrm{ReCI}$ to activate an arbitrary number of sub-carriers, leading to higher flexibility in terms of the transmission reliability and spectral efficiency than the conventional IM-OFDM-CI.

The OFDM sub-block creator takes into account $\mathbf{x}_{1}$ and $\mathbf{x}_{2}$ to generate the transmitted signal per sub-block $\mathbf{x}=$ $\left[\begin{array}{ll}\mathbf{x}_{1}^{T} & \mathbf{x}_{2}^{T}\end{array}\right]^{T}$. For each ReCI sub-block, the received signal in the frequency domain can be expressed as

$$
\mathbf{y}=\mathbf{H x}+\mathbf{n},
$$

where $\mathbf{y}=\left[\begin{array}{ll}\mathbf{y}_{1} & \mathbf{y}_{2}\end{array}\right]^{T}, \mathbf{n}=\left[\begin{array}{ll}\mathbf{n}_{1}^{T} & \mathbf{n}_{2}^{T}\end{array}\right]^{T}$, and

$$
\mathbf{H}=\left[\begin{array}{cc}
\mathbf{H}_{1} & \mathbf{0} \\
\mathbf{0} & \mathbf{H}_{2}
\end{array}\right] \text {. }
$$

The components $\mathbf{y}_{i}=\left[y_{i 1}, \ldots, y_{i N}\right]$ and $\mathbf{H}_{i}=$ $\operatorname{diag}\left\{h_{i 1}, \ldots, h_{i N}\right\}$, for $i=1,2$, are the received signal and channel matrix of the cluster $i$, respectively. The noise component is given by $\mathbf{n}_{i}=\left[n_{i 1}, \ldots, n_{i N}\right]^{T}$. The channel coefficient over each sub-carrier is represented by $h_{i \alpha}$. Each element $\left|h_{i \alpha}\right|^{2}$ is a Gamma distributed random variable with $\left|h_{i \alpha}\right|^{2} \sim \mathcal{G}(m, \beta)$, where $m$ is the distribution parameter and $\beta$ is defined as

$$
\beta=\frac{m}{\mathbb{E}\left\{\left|h_{i \alpha}\right|^{2}\right\}} .
$$

Noise on each sub-carrier follows the distribution $n_{i \alpha} \sim$ $\mathcal{C N}\left(0, N_{0}\right)$, where $N_{0}$ represents the noise variance. The average signal to noise ratio (SNR) per sub-carrier is determined by $\bar{\gamma}=\omega E_{S} / N_{0}$, where $\omega E_{s}$ denotes the average transmit power per $M$-ary modulated symbol, i.e., $\mathbb{E}\left\{|s|^{2}\right\}=$ $\omega E_{s}$. The power allocation factor and the average power per sub-carrier are respectively denoted by $\omega=N / K$ and $E_{S}$.

Consequently, the total number of information bits transmitted per sub-block is $p=p_{1}+2 p_{2}$ bits. As a result, the spectral efficiency of the ReCI system is given by

$$
\eta=\frac{\left\lfloor\log _{2}(C(N, K))\right\rfloor+2 K \log _{2} M}{2 N}[\mathrm{~b} / \mathrm{s} / \mathrm{Hz}] .
$$

In order to estimate the transmitted signal, the receiver uses an ML detector to jointly detect the indices of active sub-carriers and the corresponding $M$-ary modulated 
TABLE I

EXAMPLE OF LUT with $N=4, K=2, p_{1}=2$

\begin{tabular}{|c|c|c|c|c|c|}
\hline$p_{1}$ & $\theta$ & $\mathbf{x}_{1}^{T}$ & \multicolumn{4}{|c|}{$\mathbf{x}_{2}^{T}$} \\
\hline \hline 00 & {$[1,2]$} & {$\left[\begin{array}{llllll}a_{1}^{R}+j b_{1}^{I} & a_{2}^{R}+j b_{2}^{I} & 0 & 0\end{array}\right]$} & {$\left[\begin{array}{lllll}b_{1}^{R}+j a_{1}^{I} & b_{2}^{R}+j a_{2}^{I} & 0 & 0\end{array}\right]$} \\
\hline 01 & {$[2,3]$} & {$\left[\begin{array}{llllll}0 & a_{1}^{R}+j b_{1}^{I} & a_{2}^{R}+j b_{2}^{I} & 0\end{array}\right]$} & {$\left[\begin{array}{lllll}0 & b_{1}^{R}+j a_{1}^{I} & b_{2}^{R}+j a_{2}^{I} & 0\end{array}\right]$} \\
\hline 10 & {$[2,4]$} & {$\left[\begin{array}{llllll}0 & a_{1}^{R}+j b_{1}^{I} & 0 & a_{2}^{R}+j b_{2}^{I}\end{array}\right]$} & {$\left[\begin{array}{lllll}0 & b_{1}^{R}+j a_{1}^{I} & 0 & b_{2}^{R}+j a_{2}^{I}\end{array}\right]$} \\
\hline 11 & {$[1,3]$} & {$\left[\begin{array}{llllll}a_{1}^{R}+j b_{1}^{I} & 0 & a_{2}^{R}+j b_{2}^{I} & 0\end{array}\right]$} & {$\left[\begin{array}{llll}b_{1}^{R}+j a_{1}^{I} & 0 & b_{2}^{R}+j a_{2}^{I} & 0\end{array}\right]$} \\
\hline
\end{tabular}

symbols for both clusters. The ML detector selects the codeword that minimizes the following decision metric:

$$
\left(\hat{\theta}, \hat{\mathbf{s}}_{1}, \hat{\mathbf{s}}_{2}\right)=\arg \min _{\theta, \mathbf{s}_{1}, \mathbf{s}_{2}}\|\mathbf{y}-\mathbf{H x}\|^{2}
$$

\section{Performance Analysis}

\section{SEP Derivation}

In this section, we derive the closed-form expression for SEP of the ReCI system using ML detection. SEP is defined by the ratio of the number of error symbols to the total number of transmitted index and data symbols. Following the frame work in [10], SEP of the ReCI system is given by

$$
P_{s}=\frac{P_{I}+K P_{M}}{K+1}
$$

where $P_{I}$ and $P_{M}$ denotes the index and the $M$-ary modulated symbol error probability, respectively. We calculate SEP of the $M$-ary modulated data symbols in ReCI by using the pair-wise error probability (PEP) of modulated symbols. PEP is determined by the probability that a transmitted symbol $a_{1}$ is mistakenly detected by symbol $\hat{a}_{1}$. Particularly, the received signals per sub-carrier of two clusters are respectively rewritten as

$$
\begin{aligned}
& y_{1 \alpha}=h_{1 \alpha} x_{1 \alpha}+n_{1 \alpha}, \\
& y_{2 \alpha}=h_{2 \alpha} x_{2 \alpha}+n_{2 \alpha},
\end{aligned}
$$

where $h_{i \alpha} \sim C \mathcal{N}(0,1), n_{i \alpha} \sim C \mathcal{N}\left(0, N_{0}\right), \alpha=1, \ldots, N$, and $i=1,2$. For presentation convenience, we will ignore the sub-carrier index $\alpha$ in the following derivations. Then, we can rewrite $y_{1 \alpha}, y_{2 \alpha}$ as follows:

$$
\begin{aligned}
y_{1} & =\left(h_{1}^{R} a_{1}^{R}-h_{1}^{I} b_{1}^{I}+n_{1}^{R}\right)+j\left(h_{1}^{I} a_{1}^{R}+h_{1}^{R} b_{1}^{I}+n_{1}^{I}\right) \\
& =y_{1}^{R}+j y_{1}^{I}, \\
y_{2} & =\left(h_{2}^{R} b_{1}^{R}-h_{2}^{I} a_{1}^{I}+n_{2}^{R}\right)+j\left(h_{2}^{I} b_{1}^{R}+h_{2}^{R} a_{1}^{I}+n_{2}^{I}\right) \\
& =y_{2}^{R}+j y_{2}^{I} .
\end{aligned}
$$

As a result, we can deduce

$$
\begin{aligned}
h_{1}^{R} y_{1}^{R}+h_{1}^{I} y_{1}^{I} & =\left[\left(h_{1}^{R}\right)^{2}+\left(h_{1}^{I}\right)^{2}\right] a_{1}^{R}+h_{1}^{R} n_{1}^{R}+h_{1}^{I} n_{1}^{I} \\
& =\left|h_{1}\right|^{2} a_{1}^{R}+\bar{n}_{1}^{R}, \\
h_{2}^{R} y_{2}^{I}-h_{2}^{I} y_{2}^{R} & =\left[\left(h_{2}^{R}\right)^{2}+\left(h_{2}^{I}\right)^{2}\right] a_{1}^{I}+h_{2}^{R} n_{2}^{I}-h_{2}^{I} n_{2}^{R} \\
& =\left|h_{2}\right|^{2} a_{1}^{I}+\bar{n}_{1}^{I} .
\end{aligned}
$$

Then, we obtain

$$
\begin{aligned}
\tilde{y}_{1} & =\left(\frac{h_{1}^{R} y_{1}^{R}+h_{1}^{I} y_{1}^{I}}{\left|h_{1}\right|}\right)+j\left(\frac{h_{2}^{R} y_{2}^{I}-h_{2}^{I} y_{2}^{R}}{\left|h_{2}\right|}\right) \\
& =\left|h_{1}\right| a_{1}^{R}+j\left|h_{2}\right| a_{1}^{I}+\tilde{n}_{1}^{R}+j \tilde{n}_{1}^{I} \\
& =\left|h_{1}\right| a_{1}^{R}+j\left|h_{2}\right| a_{1}^{I}+\tilde{n}_{1},
\end{aligned}
$$

where $\tilde{n}_{1}^{R}=\bar{n}_{1}^{R} /\left|h_{1}\right|, \tilde{n}_{1}^{I}=\bar{n}_{1}^{I} /\left|h_{2}\right|$, and both $\tilde{n}_{1}^{R}$ and $\tilde{n}_{1}^{I}$ have the same distribution $\mathcal{N}\left(0, \frac{N_{0}}{2}\right)$. Thus, the distribution of $\tilde{n}_{1}=\tilde{n}_{1}^{R}+j \tilde{n}_{1}^{I}$ is given by $\tilde{n}_{1} \sim \mathcal{C N}\left(0, N_{0}\right)$. Following Equation (5) of [27], the conditional PEP of the ReCI system can be computed as follows:

$$
\begin{aligned}
P\left(a_{1} \rightarrow \hat{a}_{1} \mid h_{1}, h_{2}\right)= & \operatorname{Pr}\left[\left|\tilde{y}_{1}-\right| h_{1}\left|\hat{a}_{1}^{R}-j\right| h_{2}\left|\hat{a}_{1}^{I}\right|^{2}\right. \\
& \left.<\left|\tilde{y}_{1}-\right| h_{1}\left|a_{1}^{R}-j\right| h_{2}\left|a_{1}^{I}\right|^{2}=\left|\tilde{n}_{1}\right|^{2}\right] \\
= & Q\left(\sqrt{\frac{\left|h_{1}\right|^{2} \Delta_{R}^{2}+\left|h_{2}\right|^{2} \Delta_{I}^{2}}{2 N_{0}}}\right) \\
= & Q\left(\sqrt{\frac{\gamma_{1} \Delta_{R}^{2}+\gamma_{2} \Delta_{I}^{2}}{2}}\right)
\end{aligned}
$$

where $\Delta_{R}^{2}=\left|a_{1}^{R}-\hat{a}_{1}^{R}\right|^{2}$ and $\Delta_{I}^{2}=\left|a_{1}^{I}-\hat{a}_{1}^{I}\right|^{2}$. Assume that the average SNR is given by $\bar{\gamma}=1 / N_{0}$, let us denote $\gamma_{1}=$ $\bar{\gamma}\left|h_{1}\right|^{2}$ and $\gamma_{2}=\bar{\gamma}\left|h_{2}\right|^{2}$.

Applying the approximation of the $Q$-function [28] as $Q(t) \approx \frac{1}{12} e^{-\frac{t^{2}}{2}}+\frac{1}{4} e^{-\frac{2}{3} t^{2}}$, the average PEP of the $M$-ary modulated data symbol is obtained by

$$
P\left(a_{1} \rightarrow \hat{a}_{1}\right)=\mathbb{E}\{Q(\sqrt{\Omega})\}=\mathbb{E}\left\{\frac{1}{12} e^{-\frac{\Omega}{2}}+\frac{1}{4} e^{-\frac{2 \Omega}{3}}\right\},
$$

where $\Omega=\frac{\gamma_{1} \Delta_{R}^{2}+\gamma_{2} \Delta_{I}^{2}}{2}$. 
Using the moment generating function (MGF) for the Nakagami- $m$ fading channel [29]

$$
\mathcal{M}_{\gamma}(z)=\mathbb{E}_{\gamma}\left\{e^{-z \gamma}\right\}=\left(1-\frac{z \gamma}{m}\right)^{-m}
$$

the MGF of $\Omega$ is given by

$$
\mathcal{M}_{\Omega}(z)=\frac{1}{\left(1-\frac{\bar{\gamma} \Delta_{R}^{2} z}{2 m}\right)^{m}\left(1-\frac{\bar{\gamma} \Delta_{I}^{2} z}{2 m}\right)^{m}} .
$$

Following the MGF approach, the average PEP of the $M$-ary modulated data symbol is given by

$$
\begin{aligned}
P\left(a_{1} \rightarrow \hat{a}_{1}\right)= & \frac{\mathcal{M}_{\Omega}\left(-\frac{1}{2}\right)}{12}+\frac{\mathcal{M}_{\Omega}\left(-\frac{2}{3}\right)}{4} \\
= & \frac{1}{12} \frac{1}{\left(1+\frac{\bar{\gamma} \Delta_{R}^{2}}{4 m}\right)^{m}} \frac{1}{\left(1+\frac{\bar{\gamma} \Delta_{I}^{2}}{4 m}\right)^{m}} \\
& +\frac{1}{4} \frac{1}{\left(1+\frac{\bar{\gamma} \Delta_{R}^{2}}{3 m}\right)^{m}} \frac{1}{\left(1+\frac{\bar{\gamma} \Delta_{I}^{2}}{3 m}\right)^{m}}
\end{aligned}
$$

Using the union bound, the $M$-ary modulated symbol error probability is determined by

$$
P_{M}=\frac{1}{M} \sum_{a_{1} \in \mathcal{S}^{\phi}} \sum_{\hat{a}_{1} \neq a_{1}} P\left(a_{1} \rightarrow \hat{a}_{1}\right) .
$$

Generally, the erroneous symbol occurs when index and/or data symbols are erroneously detected at the receiver. An error index symbol causes the data symbol corresponding to the index error symbol to become erroneous. Since different clusters in the ReCI system employ a same set of active indices, this index repetition allows $P_{I}$ to achieve higher diversity gain than $P_{M}$. Therefore, SEP of the ReCI system can be approximated by

$$
P_{s} \approx \frac{K P_{M}}{K+1}
$$

\section{Asymptotic Analysis}

In the high SNR region, the average SEP of the ReCI system can be approximated by

$$
P_{s} \approx \frac{K\left((4 m)^{2 m}+3(3 m)^{2 m}\right)}{12(K+1)} \frac{1}{\left(\Delta_{I}^{2} \Delta_{R}^{2}\right)^{m}} \bar{\gamma}^{-2 m} .
$$

Remark 1: It can be seen from (22) that the ReCI system achieves the diversity order of $2 \mathrm{~m}$. Besides, the error performance depends on $K$, the reliability of the ReCI system deteriorates when $K$ increases and choosing a small $K$ allows ReCI to improve its error performance. This statement will be validated using simulation results in Section IV.

Remark 2: As can be seen from (22) that at a given $K$, SEP of ReCI is strongly influenced by the product $\Delta_{I}^{2} \Delta_{R}^{2}$. The best SEP is achieved by maximizing $\Delta=\Delta_{I}^{2} \Delta_{R}^{2}$.

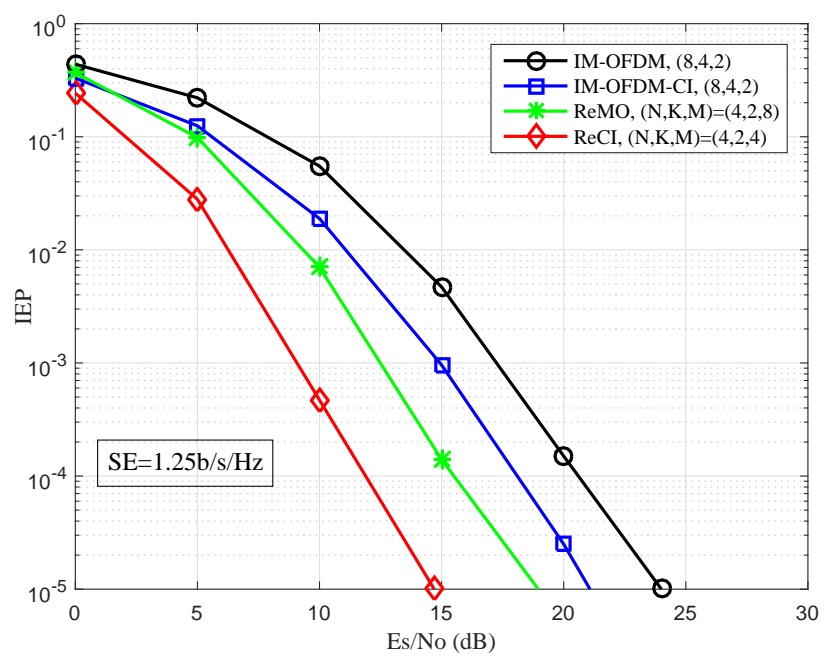

Figure 2. Index error performance of $\mathrm{ReCI}$ in comparison with the conventional IM-OFDM, IM-OFDM-CI and ReMO systems at the spectral efficiency (SE) of $1.25 \mathrm{~b} / \mathrm{s} / \mathrm{Hz}$ when $m=2, M=\{2,4,8\}, N=\{4,8\}$, $K=\{2,4\}$.

Remark 3: Based on the framework to derive BER of IMOFDM in [12], the approximated average BER of ReCI can be obtained by

$$
P_{b} \approx \frac{K P_{M}}{p_{I}+p_{c}}
$$

\section{Simulation Results and Discussions}

This section presents analytical and simulation results of the ReCI scheme in comparison with IM-OFDM [2], IM-OFDM-CI [15], and ReMO [23]. The ML detector is employed in all the addressed schemes. Assume that the channel over each sub-carrier is flat Rayleigh fading channel. For simplicity, a system configuration with $N$ subcarriers, $K$ active sub-carriers and modulation order $M$ is referred to as $(N, K, M)$.

Figure 2 compares the index error probability (IEP) of the proposed $\mathrm{ReCI}$, the conventional IM-OFDM, IM-OFDM$\mathrm{CI}$ and ReMO system at the same spectral efficiency of $1.25 \mathrm{~b} / \mathrm{s} / \mathrm{Hz}$ when $m=2, M=\{2,4,8\}, N=\{4,8\}$, $K=\{2,4\}$. It can be seen that the proposed scheme significantly improves the IEP performance of the reference systems. Particularly, at IEP of $10^{-4}$, the proposed scheme attains SNR gains of $9 \mathrm{~dB}, 5.5 \mathrm{~dB}, 3.5 \mathrm{~dB}$ over the IMOFDM, IM-OFDM-CI, and ReMO systems, respectively. Since the proposed scheme jointly uses index repetition and coordinate interleaving, it can achieve a better diversity gain in the index domain than IM-OFDM, IM-OFDMCI, and ReMO.

Figure 3 reports the SEP performance comparison between the ReCI, IM-OFDM, IM-OFDM-CI, and ReMO systems at the same spectral efficiency of $1.25 \mathrm{~b} / \mathrm{s} / \mathrm{Hz}$ 


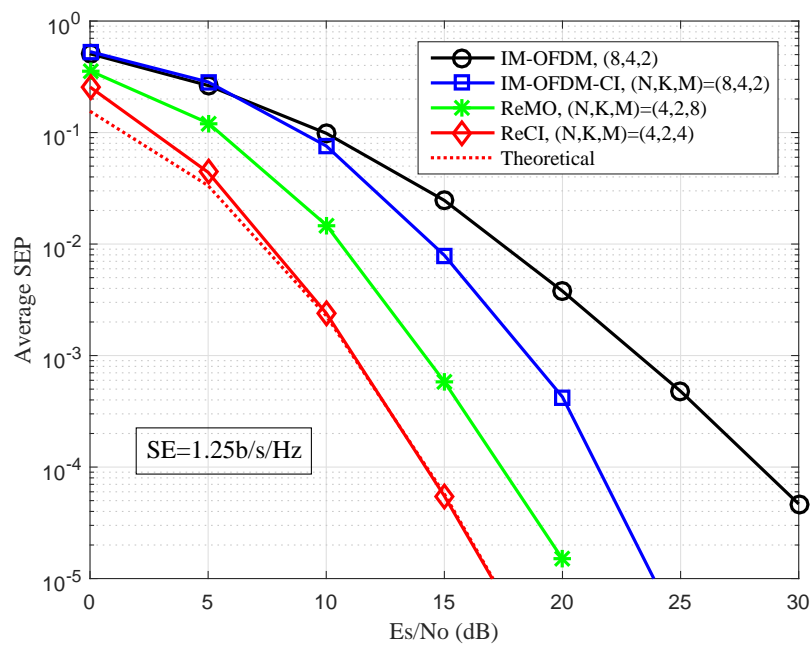

Figure 3. Comparison between the SEP performance of ReCI, IM-OFDM and IM-OFDM-CI at the spectral efficiency of $1.25 \mathrm{~b} / \mathrm{s} / \mathrm{Hz}$ when $m=2$, $M=\{2,4,8\}, N=\{4,8\}, K=\{2,4\}$.

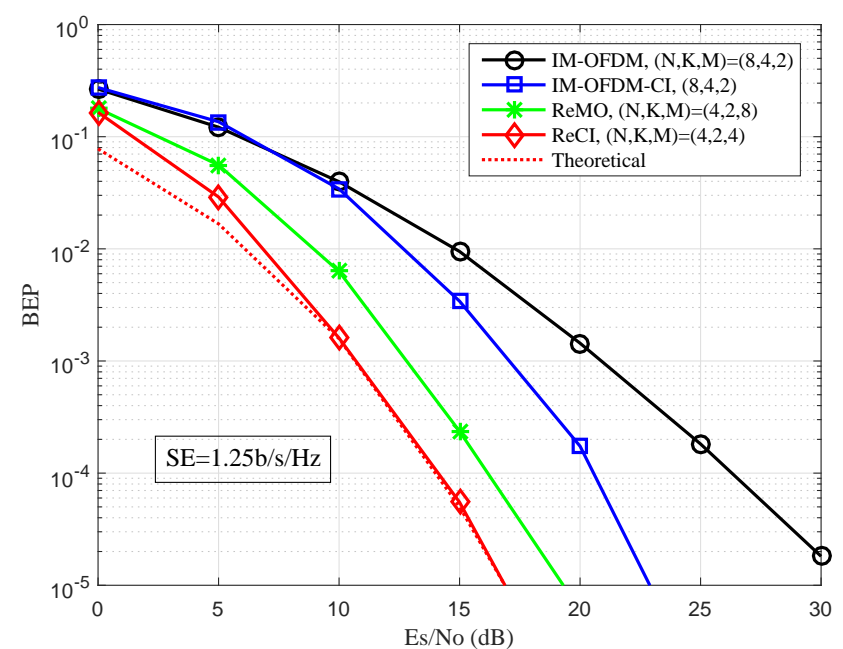

Figure 4. BEP comparison between the ReCI scheme and benchmarks at the spectral efficiency of $1.25 \mathrm{~b} / \mathrm{s} / \mathrm{Hz}$ when $m=2, M=\{2,4,8\}$, $N=\{4,8\}, K=\{2,4\}$.

when $m=2, M=\{2,4,8\}, N=\{4,8\}, K=\{2,4\}$. As seen from the figure, at the same spectral efficiency and SEP of $10^{-4}$, the ReCI scheme provides an SNR gain of about $14.5 \mathrm{~dB}, 6.5 \mathrm{~dB}$, and $2.5 \mathrm{~dB}$ over IM-OFDM, IM-OFDM-CI, and ReMO, respectively. This achievable improvement is due to the IEP improvement, as shown in Figure 2, which can reduce errors of the $M$-ary modulated symbol detection, leading to improvement in the overall error probability compared with the benchmark schemes.

Figure 4 depicts the BEP comparison between ReCI, IM-OFDM, IM-OFDM-CI and ReMO when $m=2, M=$ $\{2,4,8\}, N=\{4,8\}, K=\{2,4\}$. All considered schemes use the ML detection. As can be seen from this figure, at the

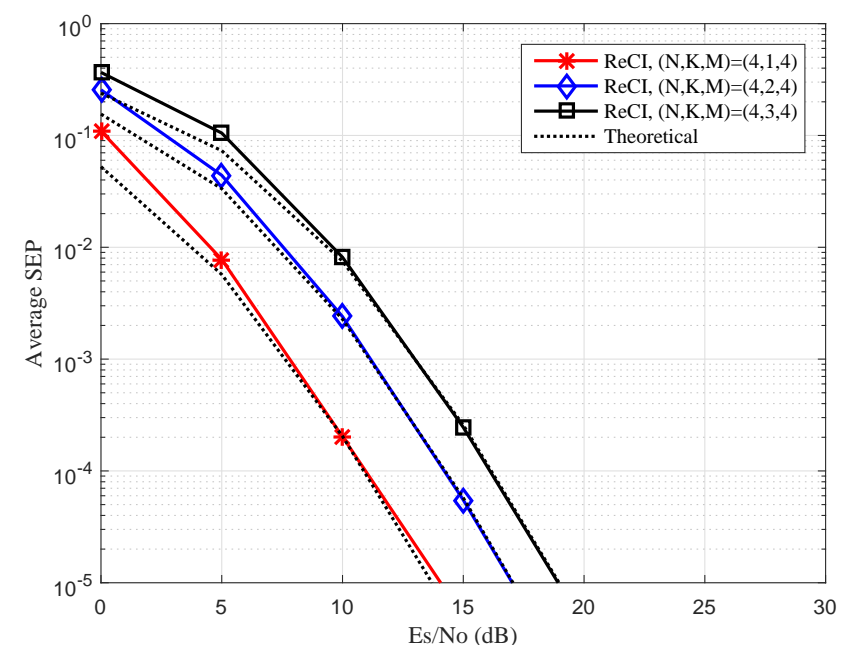

Figure 5. Impact of $K$ on the performance of ReCI when $m=2, M=4$, $N=4, K=\{1,2,3\}$.

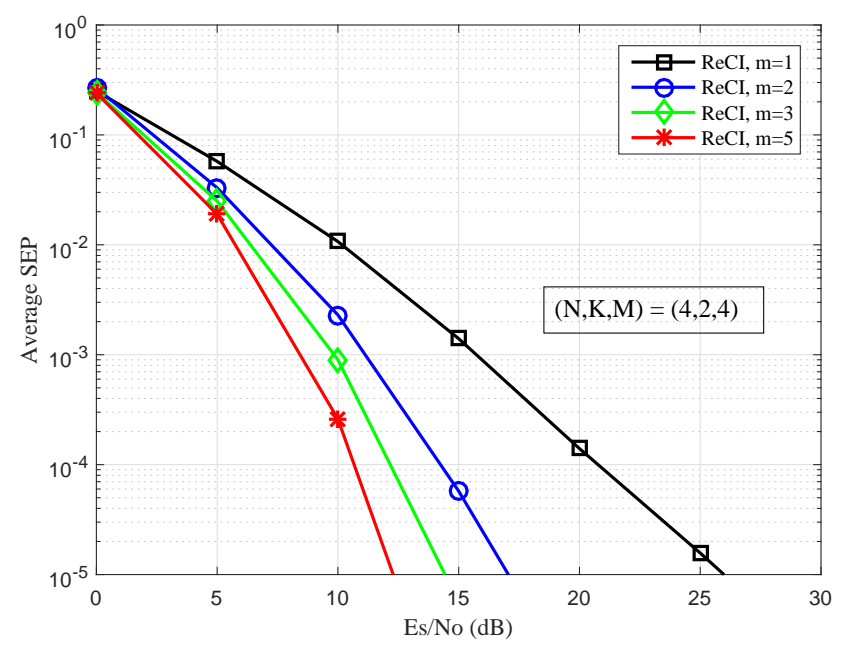

Figure 6. Impact of $m$ on the SEP performance of ReCI when $m=$ $\{1,2,3,5\}, M=4, N=4, K=2$.

same spectral efficiency of $1.25 \mathrm{~b} / \mathrm{s} / \mathrm{Hz}$, the proposed ReCI scheme achieves better BEP performance than the reference systems. For example, at BEP of $10^{-4}$, the proposed scheme can obtain SNR gains of about $13 \mathrm{~dB}, 5.5 \mathrm{~dB}$ and $1.5 \mathrm{~dB}$ over IM-OFDM, IM-ODFM-CI and ReMO, respectively. Moreover, the tightness of theoretical and simulation curves validates the accuracy of the closed-form expression for BEP derived for the proposed scheme in (23).

The accuracy of the approximate SEP expressions in (21) and (22) is validated by using the simulation results for various system parameters in Figure 5. As shown in the figure, the gap between the theoretical and simulation SEP curves are very tight in the large SNR regime for 
all system configurations. Therefore, the derived closedform expression of SEP in (21) can be used to evaluate performance of ReCI working at the high SNR regime. Moreover, it can be observed from Figure 5 that the SEP performance deteriorates as $K$ increases. Clearly, choosing $K=1$ allows $\mathrm{ReCI}$ to achieve the highest transmission reliability. Thus, it is recommended to select a small number of active sub-carriers $K$ for better system performance. This validates Remark 1.

The impact of the channel parameter $m$ on the SEP performance of $\mathrm{ReCI}$ is represented in Figure 6. It can be observed that the diversity gain of $\mathrm{ReCI}$ is strongly depended on $m$. As expected in Remark 1, when the value of $m$ increases, the system attains better SEP performance. The worst case occurs with $m=1$, which is equivalent to the Rayleigh fading channel.

\section{Conclusions}

This paper has analyzed the error performance of the repeated index modulation-OFDM with coordinated interleaving in terms of both SEP and BEP performances. The proposed scheme provides higher reliability and flexibility than the conventional IM-OFDM-CI system. By applying the same set of active sub-carrier indices in two distinguishable clusters and jointly employing the coordinate interleaving to the $M$-ary modulated symbols in them, the proposed $\mathrm{ReCI}$ scheme can improve both the index and symbol error performances while having higher flexibility between the transmission reliability and the spectral efficiency. The derived closed-form expressions gives an insight into the influence of the system parameters on the error performance. It allows to select the system configuration with better system performance. The analytical and simulation results clearly confirmed the advantages of the proposed scheme over the reference systems.

\section{REFERENCES}

[1] R. Abu-Alhiga and H. Haas, "Subcarrier-index modulation OFDM," in IEEE International Symposium on Personal, Indoor and Mobile Radio Communications. IEEE, Sep. 2009, pp. 177-181.

[2] E. Başar, Ü. Aygölü, E. Panayırcı, and H. V. Poor, "Orthogonal frequency division multiplexing with index modulation," IEEE Transactions on Signal Processing, vol. 61, no. 22, pp. 5536-5549, Aug. 2013.

[3] Y. Xiao, S. Wang, L. Dan, X. Lei, P. Yang, and W. Xiang, "OFDM with interleaved subcarrier-index modulation," IEEE Communications Letters, vol. 18, no. 8, pp. 14471450, Jun. 2014.

[4] M. Wen, X. Cheng, M. Ma, B. Jiao, and H. V. Poor, "On the achievable rate of OFDM with index modulation," IEEE Transactions on Signal Processing, vol. 64, no. 8, pp. 19191932, Apr. 2016.

[5] B. Zheng, F. Chen, M. Wen, F. Ji, H. Yu, and Y. Liu, "Low-complexity ML detector and performance analysis for
OFDM with in-phase/quadrature index modulation," IEEE Communications Letters, vol. 19, no. 11, pp. 1893-1896, Nov. 2015.

[6] T. Mao, Z. Wang, Q. Wang, S. Chen, and L. Hanzo, "Dualmode index modulation aided OFDM," IEEE Access, vol. 5, pp. 50-60, Feb. 2017.

[7] M. Wen, E. Basar, Q. Li, B. Zheng, and M. Zhang, "Multiple-mode orthogonal frequency division multiplexing with index modulation," IEEE Transactions on Communications, vol. 65, no. 9, pp. 3892-3906, May. 2017.

[8] M. Wen, B. Ye, E. Basar, Q. Li, and F. Ji, "Enhanced orthogonal frequency division multiplexing with index modulation," IEEE Transactions on Wireless Communications, vol. 16, no. 7, pp. 4786-4801, May. 2017.

[9] H. Zhang, L.-L. Yang, and L. Hanzo, "Compressed sensing improves the performance of subcarrier index-modulationassisted OFDM," IEEE Access, vol. 4, pp. 7859-7873, 2016.

[10] Y. Ko, "A tight upper bound on bit error rate of joint OFDM and multi-carrier index keying," IEEE Commun. Lett., vol. 18, no. 10, pp. 1763-1766, Oct. 2014.

[11] T. V. Luong and Y. Ko, "Symbol Error Outage Performance Analysis of MCIK-OFDM over Complex TWDP Fading," in 2017 European Wireless Conference. VDE, May 2017, pp. $1-5$.

[12] $\longrightarrow$, "A Tight Bound on BER of MCIK-OFDM With Greedy Detection and Imperfect CSI," IEEE Communications Letters, vol. 21, no. 12, pp. 2594-2597, Aug. 2017.

[13] — "Impact of CSI uncertainty on MCIK-OFDM: Tight closed-form symbol error probability analysis," IEEE Transactions on Vehicular Technology, vol. 67, no. 2, pp. 12721279, Feb. 2018.

[14] - , "Impact of Opportunistic Transmission on MCIKOFDM: Diversity and Coding Gains," in Proc. 4th EAI INISCOM. Springer, Aug. 2018, pp. 221-231.

[15] E. Basar, "OFDM with index modulation using coordinate interleaving," IEEE Wireless Communications Letters, vol. 4, no. 4, pp. 381-384, Aug. 2015.

[16] J. Zheng and R. Chen, "Achieving transmit diversity in OFDM-IM by utilizing multiple signal constellations," IEEE Access, vol. 5, pp. 8978-8988, Aug. 2017.

[17] J. Choi, "Coded OFDM-IM with transmit diversity," IEEE Transactions on Communications, vol. 65, no. 7, pp. 31643171, Jul. 2017.

[18] T. V. Luong and Y. Ko, "Spread OFDM-IM with precoding matrix and low-complexity detection designs," IEEE Transactions on Vehicular Technology, vol. 67, no. 12, pp. 11619 11 626, Dec. 2018.

[19] E. Başar, "Multiple-input multiple-output OFDM with index modulation," IEEE Signal Processing Letters, vol. 22, no. 12, pp. 2259-2263, Dec. 2015.

[20] B. Zheng, M. Wen, E. Basar, and F. Chen, "Multipleinput multiple-output OFDM with index modulation: Lowcomplexity detector design," IEEE Transactions on Signal Processing, vol. 65, no. 11, pp. 2758-2772, Jun. 2017.

[21] J. Crawford, E. Chatziantoniou, and Y. Ko, "On the SEP analysis of OFDM index modulation with hybrid low complexity greedy detection and diversity reception," IEEE Transactions on Vehicular Technology, vol. 66, no. 9, pp. 8103-8118, Apr. 2017.

[22] T. V. Luong and Y. Ko, "The BER analysis of MRC-aided greedy detection for OFDM-IM in presence of uncertain CSI," IEEE Wireless Communications Letters, vol. 7, no. 4, pp. 566-569, Aug. 2018.

[23] T. V. Luong, Y. Ko, and J. Choi, "Repeated MCIK-OFDM With Enhanced Transmit Diversity Under CSI Uncertainty," IEEE Transactions on Wireless Communications, vol. 17, no. 6, pp. 4079-4088, Jun. 2018. 
[24] T. V. Luong, Y. Ko, N. A. Vien, D. H. Nguyen, and M. Matthaiou, "Deep Learning-Based Detector for OFDMIM," IEEE Wireless Communications Letters, 2019.

[25] H. L. T. Thanh, V.-D. Ngo, M.-T. Le, and X. N. Tran, "Repeated index modulation with coordinate interleaved ofdm," in 2018 5th NAFOSTED Conference on Information and Computer Science (NICS). IEEE, Nov. 2018, pp. 114118.

[26] M. Nakagami, "The m-distribution- A general formula of intensity distribution of rapid fading," in Statistical Methods in Radio Wave Propagation. Elsevier, 1960, pp. 3-36.

[27] H. Lee, J. G. Andrews, R. W. Heath Jr, and E. J. Powers, "The performance of space-time block codes from coordinate interleaved orthogonal designs over Nakagami-m fading channels," IEEE Transactions on Communications, vol. 57, no. 3, pp. 653-664, Mar. 2009.

[28] M. Chiani, D. Dardari, and M. K. Simon, "New exponential bounds and approximations for the computation of error probability in fading channels," IEEE Transactions on Wireless Communications, vol. 2, no. 4, pp. 840-845, Jul. 2003.

[29] M. K. Simon and M.-S. Alouini, Digital communication over fading channels. John Wiley \& Sons, 2005, vol. 95.

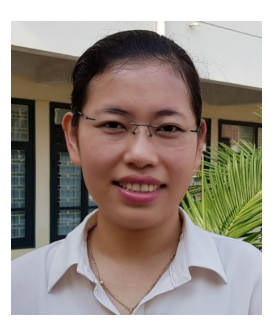

Le Thi Thanh Huyen was born in Hanoi, Vietnam in 1986. She received her B.Eng. and M.Sc. degrees both in Electronic Engineering from Le Quy Don Technical University, Hanoi, Vietnam, in 2010 and 2014, respectively. From 2010 to 2016 she worked as a lecturer at Le Quy Don Technical University. She is currently working toward her Ph.D. degree in Electronic Engineering at Le Quy Don Technical University.

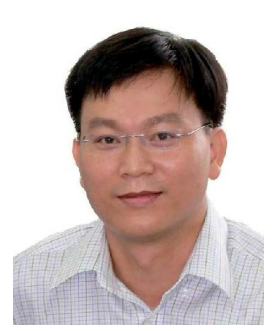

Tran Xuan Nam is currently an associate professor and head of the research group in advanced wireless communications in Le Quy Don Technical University, Hanoi, Vietnam. He received his M.Eng. in Telecommunications Engineering from University of Technology Sydney, Australia in 1998, and Dr.Eng. in Electronic Engineering from The University of Electro-Communications, Japan in 2003. From November 2003 to March 2006 he was a research associate at the University of Electro-Communications, Tokyo, Japan. Dr. Tran research interests are in the areas of space-time signal processing for communications such as adaptive antennas, space-time coding, MIMO, spatial modulation and cooperative communications. Dr. Tran is a recipient of the 2003 IEEE APS Japan Chapter Young Engineer Award, and of two best papers from the 2012 International Conference on Advanced Technologies for Communications and the 2014 National Conference on Electronics, Communications and Information Technology. He is a member of IEEE, IEICE and the Radio and Electronics Association of Vietnam. 